\title{
AVALIAÇÃO DOS EFEITOS DO TREINAMENTO EM CRIANÇAS FUTEBOLISTAS DA VILA OLIMMPICA DA MANGUEIRA
}

\author{
EVALUATION OF THE EFFECTS OF TRAINING IN CHILDREN \\ PRACTICING FIELD SOCCER FROM MANGUEIRA OLYMPIC CENTER
}

\begin{abstract}
AUTOR
Maria de Nazaré Dias Portal ${ }^{1}$

João Bittencourt da Silva ${ }^{2}$

Alan Saraiva $^{3}$

Gerson da Cruz Monte Júnior ${ }^{4}$

Ligia Gisely dos Santos Chaves ${ }^{5}$

Antonio Maria do Amaral Neto ${ }^{6}$

Antonio José da Silva?

Estélio Henrique Martin Dantas ${ }^{8}$

${ }^{1}$ Programa de Pós-Graduação Stricto Sensu

em Ciência da Motricidade Humana na

Universidade Castelo Branco - UCB/ RJ

Secretaria de Estado de Educação do Pará

[SEDUC/PA] Laboratório de Biociências

da Motricidade Humana (LABIMH - RJ)

${ }^{2}$ Programa de Pós-Graduação Stricto Sensu

em Ciência da Motricidade Humana na

Universidade Castelo Branco - UCB/RJ

Laboratório de Biociências da Motricidade

Humana (LABIMH - RJ)

${ }^{3}$ Programa de Pós-Graduação Stricto Sensu em Ciência da Motricidade Humana na

Universidade Castelo Branco - UCB/ RJ

Laboratório de Biociências da Motricidade

Humana (LABIMH - RJ)

${ }^{4}$ Programa de Pós-Graduação Stricto Sensu em Ciência da Motricidade Humana na Universidade Castelo Branco - UCB/RJ

Laboratório de Biociências da Motricidade

Humana (LABIMH - RJ)

${ }^{5}$ Programa de Pós-Graduação Stricto Sensu em Ciência da Motricidade Humana na

Universidade Castelo Branco - UCB/ RJ

Laboratório de Biociências da Motricidade Humana (LABIMH - RJ)

${ }^{6}$ Programa de Pós-Graduação Stricto Sensu em Ciência da Motricidade Humana na Universidade Castelo Branco - UCB/RJ Laboratório de Biociências da Motricidade Humana (LABIMH - RJ)

Universidade Trás os Montes

e Alto Douro - UTAD - PT

${ }^{8}$ Programa de Pós-Graduação Stricto Sensu em Ciência da Motricidade Humana na Universidade Castelo Branco - UCB/RJ Laboratório de Biociências da Motricidade Humana (LABIMH - RJ)
\end{abstract}

AVALIACÃO DOS EFEITOS

DO TREINAMENTO EM

CRIANCAS FUTEBOLISTAS DA

VILA OLÍMPICA DA MANGUEIRA 4(2): 47-53

\section{PALAVRAS-CHAVE}

treinamento; qualidades

físicas; maturação biológica.

\section{KEYWORDS}

training; physical qualities; biological maturation.

\section{RESUMO}

0 objetivo deste estudo foi avaliar o efeito de 12 semanas de treinamento nas qualidades físicas de dois grupos de crianças praticantes de futebol de campo da Vila Olímpica da Mangueira, ambos no estágio 3 de maturação biológica. As crianças foram submetidas a duas metodologias distintas durante a etapa de formação básica: a tradicional (grupo 1 - G1) e a maturacional (grupo 2 - G2). A amostra foi composta por 30 crianças do gênero masculino. Para a avaliação da Maturação Biológica foi utilizado o método de Tanner (1962). Para avaliação das qualidades físicas foram utilizados os seguintes protocolos: Coordenação (Teste de Coordenação de Burpee), Flexibilidade (Testes Angulares de Goniometria], Força dinâmica (Teste de Preensão Manual de Dinamometria), Força explosiva (Teste de Impulsão Vertical) e Velocidade (Teste de velocidade de $\mathbf{3 0}$ metros lançado). 0 procedimento estatístico utilizado foi o Teste-t de Student para amostras independentes e repetidas com um nível de significância de $p \leq 0,05$. Quando se comparou o G1 com o G2 observaram-se os seguintes resultados do $\Delta \%$ e do p-valor, respectivamente: coordenação ( $\Delta \%=14,11 \% ; p=0,00)$, força dinâmica $(\Delta \%=3,01 ; p=0,49)$ e força explosiva ( $\Delta \%=1,03 ; p=0,63$ ), flexibilidade $(\Delta \%=4,39 \% ; p=0,00$ ), resistência aeróbica $(\Delta \%=0,91 \% ; p=0,00)$ e velocidade $(\Delta \%=-0,96 ; p=0,00)$. Os resultados permitiram concluir que a utilização de uma metodologia levando em consideração a maturação biológica se mostrou mais eficiente no desenvolvimento de qualidades físicas de infantes no estágio 3 de maturação, praticantes de futebol de campo na Vila Olímpica da Mangueira.
ABSTRACT

The aim of this study was to evaluate the differences, found after 12 weeks of training, in the development of the physical qualities of two groups of children practicing soccer at Mangueira Olympic Center, Both groups were in the third stage of biological maturation, and were submitted to two different methodologies, during the stage of basic formation: the traditional and the maturational. The sample was composed by 30 male infants. For the evaluation of the biological maturation the board of pictures" by Tanner was used (1962), for the self evaluation protocol proposed by Matsudo \& Matsudo (1991). For the evaluation of the physical qualities the following protocols were used: coordination (test of coordination of Burpee), flexibility (angular goniometric test), dynamic power (test of manual dynamometric pressure), explosive power (test of vertical impulse) and speed (speed test of 30 meters sprint). The statistic procedure used was the Student's t-test and Student's t-test (independent protocol for inter-groups analysis]. The study established $\mathrm{p} \leq 0.05$ to statistic significance. When we compared G1 with G2 observed the results do $\Delta \%$ and p-valor, respectively: coordination $(\Delta \%=2,91 \%$; $p=0,00)$, dynamic power $(\Delta \%=3,01$; $p=0,49)$ and explosive power $(\Delta \%=1,03$; $p=0,63)$, flexibility $(\Delta \%=4,39 \% ; p=0,00)$, aerobic resistance $(\Delta \%=0,91 \% ; p=0,00)$ and speed $(\Delta \%=-0,96 ; p=0,00)$. The results allowed concluding that the experimental group, which used the maturational methodology, showed significant statistic differences in the variables coordination, flexibility, aerobic resistance and speed compared to the traditional group. 


\section{INTRODUC̣̃̃O}

A aptidão física tem componentes motoras e de saúde relacionados. A participação em atividades físicas durante a infância pode ajudar ao desenvolvimento de habilidades motoras e incutir o hábito saudável, especialmente cardiovascular ${ }^{13,22,23}$. 0 esporte é um dos maiores fenômenos sociais e um dos principais meios para a cultura de paz, baseado numa ciência de sustentação entre o homem e a sociedade por enfatizarem a qualidade de vida e o entretenimento. Pesquisas tem claramente determinado que o exercício é a chave contribuinte da saúde ${ }^{21}$.

Usufruir apenas de informação proveniente da idade cronológica, é sintetizar ao máximo a complexidade que envolve esse período do crescimento, desenvolvimento e maturação, e as possíveis relações com o desempenho motor e aptidão física? ${ }^{2}$.

Constata-se que jovens (futebolistas ou não] de mesma idade e género, apresentam diferenças significativas ao nível dos aspectos somáticos, de aptidão geral e específica, e respectivas habilidades ${ }^{16}$. Diversos autores $^{1,9,10,11,14}$ procuraram associar e recomendar o treinamento e desenvolvimento das qualidades físicas a programas sistemáticos de acordo com os níveis de maturação biológica.

Assim, pode se dizer que para o pleno desenvolvimento do jovem atleta, se deve investigar o processo de crescimento e desenvolvimento (genótipo e fenótipo), produtores de expressões quantitativas $e$ qualitativas que implicam uma série de transformações biomecânicas, fisiológicas, psicológicas e socioculturais.
Mais do que nunca atletas novos estão treinando intensivamente e competem em níveis elevados em um período associado com muitas mudanças no crescimento. Estudos longitudinais de maturação são importantes para elucidar o desenvolvimento de parâmetros físicos, além do mais, a puberdade nos seres humanos é caracterizada por grandes mudanças hormonais tendo por resultado a maturação física e a maturação sexual ${ }^{10,11}$.

Assim, o presente estudo tem por objetivo avaliar a diferença encontrada após 12 semanas de treinamento, no desenvolvimento das qualidades físicas de dois grupos de infantes praticantes de futebol de campo da Vila Olímpica da Mangueira, ambos no estágio 3 de maturação biológica, submetidos a duas metodologias distintas durante a etapa de formação básica: a tradicional (grupo 1) e a maturacional [grupo 2].

\section{MATERIAIS E MÉTODOS}

\section{Amostra}

Para este estudo a amostra foi selecionada de forma intencional e constituída de 30 infantes do gênero masculino, voluntários praticantes de futebol de campo da Vila Olímpica da Mangueira.

Na tabela 1 encontram-se os resultados descritivos do $\mathbf{G 1}$, onde se observa que as variáveis estatura e massa corporal apresentaram baixa dispersão ( $C V<25 \%$ ), tendo a média como melhor medida de tendência central, entretanto para as variáveis referentes ao somatótipo dos indivíduos, que apresentaram alta dispersão, esta seria a mediana. As variáveis: idade e endomorfia não seguiram a distribuição Normal $(p<0,05)$.
Na tabela 2 estão os resultados descritivos do G2, onde se observa que as variáveis referentes ao somatótipo dos indivíduos apresen.taram alta dispersão (CV > 25\%), portanto a mediana é a melhor medida de tendência central. As variáveis: idade e endomorfia não seguiram a distribuição Normal $(p<0,05)$.

\section{Procedimentos}

Para a caracterização da amostra foi realizada a verificação do somatotipo de Heath \& Carter (1990): tomada de massa corporal e estatura corporal (balança com estadiômetro FILIZOLA - BRASIL), medição das pregas subcutâneas tricipital, supraespinhal, subscapular e geminal medial (compasso de dobras cutâneas - CESCORF - BRASIL], medição dos perímetros do braço flexionado em contração máxima e da panturrilha medial (fita antropométrica - SANNY - BRASIL], e avaliação dos diâmetros bi-epicondilar de úmero e bi-epicôndilo femoral (paquímetro - CARDIOMED - BRASIL). Para avaliação da maturação sexual foi utilizado o método de Tanner (1962), através do protocolo de auto-avaliação das características sexuais secundárias, proposto por Matsudo (1991). A auto-avaliação foi realizada em sala apropriada, feitas individualmente e na presença do professor.

Para avaliar as qualidades físicas foram realizados os seguintes protocolos: Coordenação (Teste de Coordenação de Burpee/cronômetro da marca Polar], Flexibilidade (Testes Angulares de Goniometria, dos seguintes movimentos: Extensão Horizontal do Ombro, Flexão da Articulação do Ombro, Abdução da Articulação do Ombro, Flexão da 
Coluna Lombar, Extensão do Quadril, Abdução da Coxa/Goniômetro da marca "Lafayette Goniometer Set" e colchonete Hoorn-Brasil], Força dinâmica (Teste de Preensão Manual de Dinamometria/Dinamômetro da marca Jamar-Canadá), Força explosiva (Teste de Impulsão Vertical/Tábua de $\mathbf{1 . 5 0} \mathrm{m}$ de comprimento e $30 \mathrm{~cm}$ de largura) e Velocidade (Teste de velocidade de $\mathbf{3 0}$ metros lançado/Área útil de aproximadamente $\mathbf{5 0}$ metros e dois cronômetros da marca Polar]. Os dois grupos experimentais realizaram o treinamento durante 12 semanas, 2 vezes por semana, com a duração de 60 minutos por sessão de aula, totalizando 24 aulas de intervenção, que foram prescritas de acordo com a metodologia utilizada. $\mathbf{O}$ grupo 1 realizou a Metodologia de Formação Maturacional [G1], que é a metodologia proposta neste estudo, onde no momento do planejamento do treinamento, adapta as atividades a serem desenvolvidas de acordo com o estágio maturacional e não somente a idade cronológica, enquanto que o grupo 2 utilizou a Metodologia Tradicional (G2), que consideramos como a metodologia utilizada pelo professor local, que leva em consideração apenas a idade biológica no momento do planejamento das atividades a serem desenvolvidas.

\section{Estatística}

0 tratamento estatístico foi composto pela análise descritiva, (média, mediana, desvio-padrão, coeficiente de variação, erro padrão da média]. A análise inferencial foi realizada através do teste de Shapiro-Wilk, para verificar a homogeneidade da amostra. 0 procedimento estatístico do Teste-t de Student, pareado

\begin{tabular}{lccccc}
\hline Variáveis & x/sd & Md & E & CV & p-valor \\
\hline Idade & $10,86 \pm 1,06$ anos & 711,00 & 0,27 & 9,76 & 0,00 \\
Massa corporal & $39,84 \pm 6,68 \mathrm{~kg}$ & 39,00 & 1,72 & 16,76 & 0,12 \\
Estatura & $147,20 \pm 10,12 \mathrm{~cm}$ & 146,00 & 2,60 & 6,87 & 0,96 \\
Endomorfia & $2,70 \pm 1,51$ & 2,06 & 0,39 & 55,92 & 0,03 \\
Mesomorfia & $3,57 \pm 1,15$ & 3,62 & 0,29 & 32,21 & 0,88 \\
Ectomorfia & $3,08 \pm 1,21$ & 2,93 & 0,31 & 39,29 & 0,78 \\
\hline
\end{tabular}

TABELA1

Resultados descritivos do G1.

$\mathrm{X}$ = média; $\mathbf{s d}$ = desvio padrão; $\mathbf{M d}=$ mediana; $\mathrm{E}=$ erro padrão da média; CV = coeficiente de variação; SH = Teste de Shapiro-Wilk; p<0,05.

\begin{tabular}{lccccc} 
Variáveis & $\mathbf{x} / \mathbf{s d}$ & Md & $\mathbf{E}$ & $\mathbf{C V}$ & p-valor \\
\hline Idade & $11,03 \pm 0,81$ anos & 11,00 & 0,21 & 7,14 & 0,04 \\
Massa corporal & $40,44 \pm 7,66 \mathrm{~kg}$ & 38,70 & 1,97 & 18,94 & 0,05 \\
Estatura & $147,33 \pm 9,19 \mathrm{~cm}$ & 145,00 & 2,37 & 6,23 & 0,36 \\
Endomorfia & $2,57 \pm 1,34$ & 2,18 & 0,34 & 52,14 & 0,00 \\
Mesomorfia & $3,51 \pm 1,24$ & 3,62 & 0,32 & 35,32 & 0,06 \\
Ectomorfia & $2,99 \pm 1,15$ & 3,05 & 0,29 & 38,46 & 0,99 \\
\hline
\end{tabular}

TABELA2

Resultados descritivos do G2.

$\mathrm{X}$ = média; $\mathbf{s d}=$ desvio padrão; $\mathbf{M d}=$ mediana; $\mathrm{E}$ = erro padrão da média; CV = coeficiente de variação; SH = Teste de Shapiro-Wilk; p<0,05.

\begin{tabular}{|c|c|c|c|c|}
\hline \multirow[t]{2}{*}{ Variáveis } & \multicolumn{2}{|c|}{$x / s d$} & \multirow[t]{2}{*}{$\mathbf{t}$} & \multirow[t]{2}{*}{ p-valor } \\
\hline & Pré & Pós & & \\
\hline Coordenação & $4,33 \pm 0,61$ & $5,46 \pm 0,51$ & $-12,47$ & 0,00 \\
\hline Força dinâmica & $19,46 \pm 3,90$ & $21,53 \pm 3,90$ & $-10,02$ & 0,00 \\
\hline Força explosiva & $26,40 \pm 3,81$ & $29,93 \pm 3,61$ & $-18,41$ & 0,00 \\
\hline Flexibilidade & \multicolumn{2}{|c|}{$1,04 \pm 0,01$} & & \\
\hline Resistência Aeróbica & $37,20 \pm 2,29$ & $38,28 \pm 2,05$ & $-9,15$ & 0,00 \\
\hline Velocidade & $7,87 \pm 0,75$ & $7,48 \pm 0,73$ & 8,53 & 0,00 \\
\hline
\end{tabular}

TABELA3

Resultados do Teste-t de Student pareado para o G1.

$X=$ média; sd $=$ desvio padrão; $E=$ erro padrão da média; $\Delta \%=$ variação percentual; $\Delta$ = variação absoluta; $\mathrm{t}=$ Teste-t de Student; $\mathrm{p} \leq 0,05$. 


\begin{tabular}{lccccc}
\hline \multirow{2}{*}{ Variáveis } & \multicolumn{3}{c}{ x/sd } & t & p-valor \\
\cline { 2 - 3 } & \multicolumn{2}{c}{ Pré } & Pós & \\
\hline Coordenação & $4,06 \pm 0,25$ & $4,60 \pm 0,56$ & & $-4,00$ & 0,00 \\
Força dinâmica & $19,13 \pm 2,79$ & $20,66 \pm 2,79$ & $-11,50$ & 0,00 \\
Força explosiva & $26,06 \pm 3,30$ & $29,33 \pm 3,30$ & $-15,83$ & 0,00 \\
Flexibilidade & \multicolumn{1}{c}{$1,00 \pm 0,02$} & & \\
Resistência Aeróbica & $33,37 \pm 3,71$ & $34,06 \pm 3,71$ & $-4,94$ & 0,00 \\
\hline Velocidade & $6,80 \pm 0,33$ & $6,39 \pm 0,31$ & 5,37 & 0,00 \\
\hline
\end{tabular}

TABELA4

Resultados do Teste-t de Student pareado para o G2.

$X$ = média; sd = desvio padrão; $E$ = erro padrão da média; $t$ = Teste-t de Student; $p \leq 0,05$.

\begin{tabular}{|c|c|c|c|c|}
\hline \multirow{2}{*}{ Variáveis } & \multicolumn{2}{|c|}{ Média/sd } & \multirow[t]{2}{*}{$\mathbf{t}$} & \multirow[t]{2}{*}{ p-valor } \\
\hline & G1 & G2 & & \\
\hline Coordenação & $4,33 \pm 0,61$ & $4,06 \pm 0,25$ & 1,54 & 0,13 \\
\hline Força dinâmica & $19,46 \pm 3,90$ & $19,13 \pm 2,79$ & 0,26 & 0,79 \\
\hline Força explosiva & $26,40 \pm 3,81$ & $26,06 \pm 3,30$ & 0,25 & 0,80 \\
\hline Flexibilidade & $1,04 \pm 0,01$ & $1,00 \pm 0,02$ & 0,12 & 0,00 \\
\hline Resistência Aeróbica & $37,20 \pm 2,29$ & $33,36 \pm 3,71$ & 3,40 & 0,02 \\
\hline Velocidade & $7,87 \pm 0,75$ & $6,80 \pm 0,33$ & 5,07 & 0,00 \\
\hline
\end{tabular}

\section{TABELA5}

Resultados do Teste-t independente entre G1 e G2 antes da intervenção.

$\mathrm{X}=$ média; $\mathbf{s d}=$ desvio padrão; $\mathrm{E}=$ erro padrão da média; $\mathrm{t}=$ Teste-t de Student; $\mathrm{p} \leq 0,05$.

\begin{tabular}{lcccc} 
Variáveis & $\Delta \%[\mathrm{G} 1 / \mathrm{G} 2)$ & $\Delta \%[\mathrm{G}$ ] $/ \mathrm{G} 2)$ & $\mathrm{t}$ & p-valor \\
\hline Coordenação & $14,11 \%$ & $2,05 \mathrm{seg}$ & 4,63 & 0,00 \\
Força dinâmica & $3,01 \%$ & $1,35 \mathrm{~kg}$ & 0,69 & 0,49 \\
Força explosiva & $1,03 \%$ & $1,08 \mathrm{~m}$ & 0,47 & 0,63 \\
Flexibilidade & $4,39 \%$ & $26,82 \mathrm{gr}$ & 6,86 & 0,00 \\
Resistência Aeróbica & $0,91 \%$ & $1,38 \mathrm{seg}$ & 3,84 & 0,00 \\
Velocidade & $-0,96 \%$ & $0,84 \mathrm{seg}$ & 5,29 & 0,00 \\
\hline
\end{tabular}

\section{TABELA6}

Resultados do Teste-t independente entre G1 e G2 após a intervenção.

$\Delta \%[$ (G1/G2] = variação percentual; $\Delta$ [G1/G2] = variação absoluta

(média grupo maturação/média grupo tradicional); $t$ = Teste-t independente; $p<0,05$. foi utilizado para verificar as diferenças entre as médias das qualidades físicas antes e depois da aplicação do procedimento experimental. $O$ segundo passo consistiu na comparação dos valores médios dos respectivos grupos, tendo como protocolo estatístico o teste t de Student (protocolo independente na ótica da análise inter-grupo]. 0 estudo admitiu o nível de $p \leq 0,05$ para a significância estatística. Foi utilizado como ponto de corte o CV de $25 \%$ para definição de qual meIhor medida de tendência central.

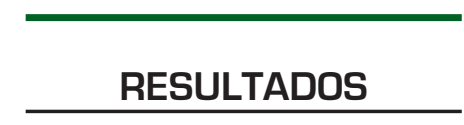

Na tabela 3, observam-se os resultados do Teste-t de Student pareado para os valores médios das variáveis referentes às qualidades físicas analisadas no G1. Constata-se que todas as variáveis apresentaram valores médios estatisticamente significativos após o período de tempo de 12 semanas.

Na tabela 4, observam-se os resultados do Teste-t de Student para amostras emparelhadas, para os valores médios das variáveis referentes às qualidades físicas analisadas no G2. Constata-se que todas as variáveis apresentaram diferenças significativas.

Na tabela 5 encontram-se os resultados do Teste-t de Student para amostras independentes. $\mathbf{O}$ mesmo consistiu na comparação dos valores médios dos respectivos grupos, antes do período de intervenção. Observa-se que apenas a variável velocidade apresentou diferença significativa quando comparados os valores médios entre os grupos, ou seja, os grupos seguiram uma distribuição normal, portanto eram homogêneos. 
Na tabela 6 encontram-se os resultados do Teste-t de Student para amostras independentes após o período de intervenção. Observa-se que as variáveis: coordenação, flexibilidade e velocidade apresentaram diferenças significativas quando comparados os valores médios entre os grupos. Também se apresentam os valores percentuais médios em cada variável antes e após a intervenção dentro de cada grupo. Finalmente, constatam-se a razão entre a melhoria alcançada pelos grupos maturacional e tradicional, respectivamente.

\section{DISCUSSÃO DOS RESULTADOS}

Após a avaliação da somatotipia, a amostra do $\mathbf{G 1}$ foi classificado como mesomorfo-ectomorfo, corroborando com os estudos de Seabra et al. ${ }^{19}$ e o grupo 2 como mesomorfo balanceado. Segundo Alonso ${ }^{1}$, estes dados evidenciam que a predominância do componente mesomorfia seguido pela ectomorfia e endomorfia caracteriza em especial a primeira aceleração de crescimento seguida de ganho de massa muscular, além disso, Malina et al. ${ }^{15}$ observam um aumento de possibilidades de maturação acelerada em grupos de crianças com o componente mesomórfico predominante.

Quando se realizou a avaliação intragrupos do grupo 1 e do grupo 2 , notou-se que todas as variáveis apresentaram diferenças significativas, no entanto o grupo 1 apresentou valores médios superiores ao grupo 2 , exceto na variável velocidade.

A velocidade e a coordenação aumentam progressivamente entre os estágios maturacionais 1, 2, e 3 e relacionam a progressão dessas qualidades físicas com o aumento da massa corporal, estado maturacional e estatura. Na presente pesquisa, a melhora da velocidade, ganhos significativos, podem estar correlacionados à melhora significativa da coordenação durante este período da puberdade ${ }^{1,3,24}$.

Diversos autores ${ }^{6,7,26}$ verificaram que com o aumento da idade, o nível de maturação sexual também irá aumentando e a força explosiva pode também apresentar um aumento significativo de acordo com a maior maturação sexual, ocorrendo uma grande variação de força de preensão (dinamometria), com o aumento dos níveis de maturação sexual. Na presente pesquisa houve melhora significativa desta variável apenas na avaliação intragrupos. Sugere-se que na intervenção para este movimento, os exercícios não tenham sido adequados ou o tempo de execução não provocou adaptação na articulação treinada (membros superiores], visto que os infantes são praticantes de futebol de campo. Quanto à variável força explosiva apesar das diferenças estatisticamente significativas encontradas terem sido na avaliação intragrupos, os resultados corroboram com outros estudos de diversos autores $^{5,8,15,25}$ quando crianças com a maturação avançada tendem a ter melhor desempenho nos testes de força, potência e velocidade, quando comparados com os que estão com a maturação normal ou na maturação atrasada na mesma idade, visto que na presente pesquisa, o grupo apresentou a idade média de 11,10 anos mostrando a precocidade na maturidade.

Quanto à variável Resistência Aeróbica as diferenças estatisticamente significativas encontradas, corroboram com estudos de outros autores $^{4,12,17,20}$ que afirmam que as respostas cardiovasculares e pulmo- nares ao exercício modificam-se com a idade e funcionam de forma diferenciada na criança e no adolescente em relação ao adulto, no entanto as alterações mais aparentes estão mais relacionadas à massa corporal ou ao estado de maturação biológica. 0 V02máx absoluto aumenta durante o crescimento devido ao aumento da quantidade de massa muscular ativa durante a atividade, o que sugere ser este um bom indicador desta variável, pois crianças em maior nível maturacional tendem a ter um maior V02 de pico. Corroboram ainda com estes achados a afirmação de Faingenbaum et al. e Sallet et al $^{7,18}$ que relatam que pesquisas da década passada fornecem evidências que as crianças podem aumentar sua força de músculo acima e além do crescimento e da maturação participando em um programa de treinamento de resistência, e que além da melhoria da força, ocorrem melhorias nas habilidades do desempenho do motor, na aptidão cardiorespiratória, na densidade mineral do osso, e na composição do corpo. Alguns autores ${ }^{22,25,26}$ afirmam que o exercício físico é um estimulo potente para mudanças hormonais nas crianças e nos adultos, entretanto, não há nenhuma evidência que os processos do crescimento e de puberdade estão influenciados pelo treinamento.

Vale ressaltar que quando se comparou as duas metodologias de treinamento utilizadas neste estudo observou-se que o G1 apresentou diferenças estatisticamente significativas para as variáveis coordenação e flexibilidade, no entanto nas demais variáveis, exceto na velocidade, apesar das diferenças não serem significativas, os valores médios foram superiores também para o G1. 


\section{CONCLUSÃO}

É difícil comparar os resultados desta pesquisa, os efeitos dos protocolos selecionados para a avaliação da intervenção por causa das diferenças no programa, duração do estudo e metodologias do treinamento utilizadas.

Mas os resultados deste estudo mostraram que a utilização da Metodologia de Formação Maturacional se mostrou mais eficiente no desenvolvimento de qualidades físicas de infantes no estágio 3 de maturação, praticantes de futebol de campo na Vila Olímpica da Mangueira.

Faz-se necessário inicialmente levar o maior número possível de crianças à prática de atividades esportivas, de forma a experimentarem atividades motoras generalizadas caracterizadas pelas diversas modalidades de esportes, levando em consideração sua idade biológica.

Para crianças, estimular a prática de atividades esportivas pode ajudar a desenvolver uma atitude positiva em torno do corpo, tornando-os conscientes para que tenham motivação de incorporar a atividade física no seu hábito de vida.

É importante que profissionais possam propiciar à criança experiências variadas, respaldados em metodologias científicas adequadas e sistematizadas, contínuas e progressivas, facilitando dessa forma o desenvolvimento ótimo de suas potencialidades.

\section{CORRESPONDÊNCIA}

Maria de Nazaré Dias Portal

Avenida Pedro Miranda nํ 638 Pedreira - Belém/Pa.

CEP: 66.085-005

\section{REFERÊNCIAS}

1. Alonso L, Silva Dantas P; Fernandes Filho J (2005). Characteristics of sexual maturation, dermatoglifics, somatotype and physical qualities um futsal young athletes. Fiep Bulletin v.16: 3-40.

2. Bojikian P, Massa M, Martin RHC, Teixeira CP, Kiss MAPDM, Böeme MTS (2002). Auto avaliação puberal feminina por meio de desenhos e fotos. Rev Bras Ativ Fís Saúde 12(2): 24-29.

3. Borges FS, Matsudo SMM, Matsudo VKR (2004). Perfil antropométrico e metabólico de rapazes pubertários da mesma idade cronológica em diferentes níveis de maturação sexual. Rev. Bras. Cienc. Mov. 12[4]: 7-12.

4. Chamari K, Hachana Y, Kaouech F, Jeddi R, Moussa-Chamari I And Wisloff $U$ (2005). Endurance training and testing with the ball in young elite soccer players. Brit. Jour Spor Med. 39: 24-28.

5. Eisenmann JC, Malina RM (2003). Age and sex-associated variation in neuromuscular capacities of adolescent distance runners. Journal Sport Sci. England, 21(7):551-7.

6. Faigenbaum AD, Milliken LA And Westcott WL (2003). Maximal strength testing in healthy children. Journal of Strength Conditioning Research. 17(1):162-166.

7. Faigenbaum AD, Loud RL, O'connell J, Glover S, O'connell J And Westcott WL (2001). Effects of Different Resistance Training Protocols on Upper-Body Strength and Endurance Development in Children. Journal of Strength and Conditioning Research. 15(4), 459-465.

8. Gabbett TJ (2002). Physiological characteristics of junior and senior rugby league players. $\mathrm{Br}$. J. Sports Med. 36:334-339.
9. Georgopoulos NA, Theodoropoulou A, Leglise M, Vagenakis AG And Markou KB (2004). Growth and Skeletal Maturation in Male and Female Artistic Gymnasts. The Journal of Clinical Endocrinology \& Metabolism. 89(9):4377-4382.

10. Gurd B, Klentrou P [2003]. Physical and pubertal development in young male gymnasts. J Appl Physiol. 95:1011-1015.

11. Hansen L, Klausen K (2004). Development of aerobic power in pubescent male soccer players related to hematocrit, hemoglobin and maturation. A longitudinal study. Journal of Sports Medicine \& Physical Fitness. 44[3]:219-23.

12. Jan J. Kaczor, Wieslaw Ziolkowski, Jerzy Popinigis, And Mark A. Tarnopolsky (2005). Anaerobic and Aerobic Enzyme Activities in Human Skeletal Muscle from Children and Adults. Pediatric Research. 57:3.

13. Koutedakis Y, Bouziotas C (2003). National physical education curriculum: motor and cardiovascular health related fitness in Greek adolescents. Br. J. Sports Med. 37;311-314.

14. Malina RM. Maturated associated variation in the growth and functional capacities of Youth Football (soccer) Players 13-15 years. (2004) European Journal of Applied Physiology. 91:(5-6) 555-562.

15. Malina RM, Cumming SP, Morano PJ, Barron M, Miller S (2005). Maturatity Status of Youth Football Players: A Noninvasive Estimate. Medicine \& Science in Sports \& Exercise, 37:(6)1044-1052.

16. Matsudo VKR, Matsudo SM (1991). Validade da Auto-avaliação na Determinação da Maturação Sexual. Revista Brasileira de Ciência e Movimento. 5:(2)18-35. 
17. Effects of Resistance Training on protein utilization in in healthy children. Medicine Science Sports Exercise. 34:(5)820-827.

18. Sallet P, Perrier D, Ferret JM, Vitelli V, Baverel G (2005). Physiological differences in professional basketball players as a function of playing position and level of play. Journal of Sports Medicine \& Physical Fitness. 45(3):291-4.

19. Seabra A, Maia JA, Garganta R (2001). Crescimento, maturação e aptidão física, forma explosiva e habilidades motoras específicas. Estudo em jovens futebolistas e não futebolistas do sexo masculino dos 12 aos 16 anos de idade. $R e$ vista Portuguesa de Ciências do Desporto. 1(2):32-35.

20. Silva RJS (2003). Capacidades Físicas e os testes motores voltados à promoção da saúde em crianças e adolescentes. Revista Brasileira de Cineantropometria \& Desempenho Humano. 5(1).

21. Stubbe JH, Boomsma DI, De Geus EJC (2005). Sports Participation during Adolescence: A Shift from Enviromental to Genetic Factors. Medicine \& Science in Sports \& Exercise. 37(4):563-570.
22. Suris JC, Parrera N (2005). Don't stop, don't stop: physical activity and adolescence. International Journal of Adolescent Medicine and Healthy. 17(1):67-78.

23. Tammelin T (2005). A review of longitudinal studies on youth predictors of adulthood physical activity. International Journal of Adolescent Medicine and Healthy. 17 (1):3-12.

24. Telama R et al. (2005). Physical Activity from childhoo to adulthood: a 21-year tracking study. American Journal of Preventive Medicine. 28 (3):267-73.

25. Tsolakis C, Vagenas G, Dessypris A (2003). Growth and anabolic hormones, leptin, and neuromuscular performance in moderately trained prepubescent athletes and untrained boys. Journal of Strength and Conditioning Research, 17(1): :40-46.

26. Tsolakis C, Messinis D, Stergioulas A And Dessypris A (2000). Hormonal responses after strength training and detraining in prepubertal and pubertal boys. Journal Strength Conditioning Research. 14(3): 26-32.

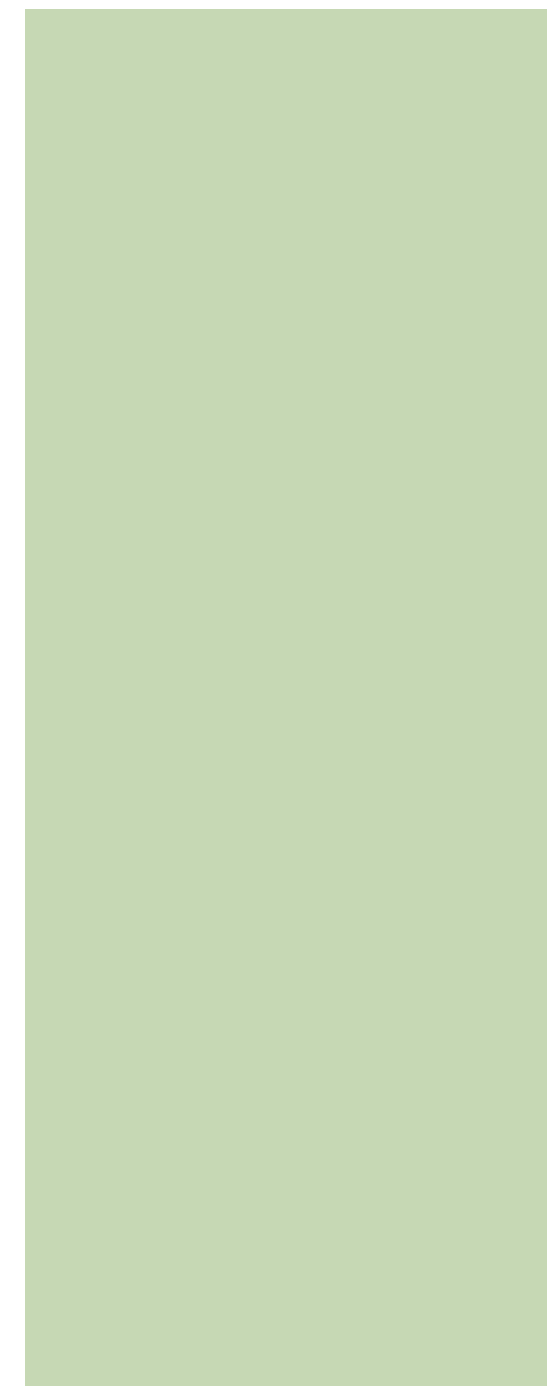

